\title{
CONVERGENCE ANALYSIS OF SOME FACTORIZATION ITERATIVE METHODS FOR M-MATRICES
}

\author{
R. Beauwens \\ Northwestern University \\ Evanston, Illinois
}

October 1973

PREPARED FOR THE U. S. ATOMIC ENERGY COMMISSION UNDER CONTRACT NO. AT $(11-1)-2280$

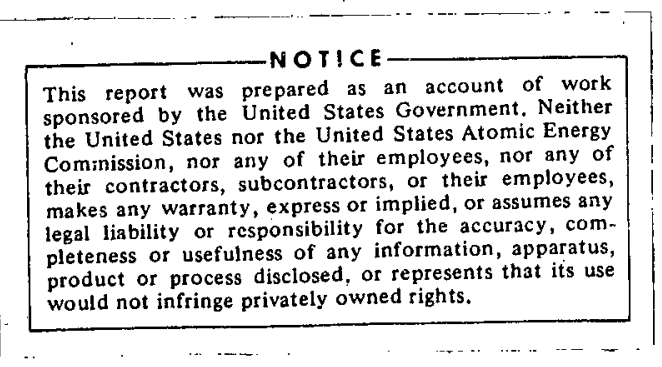




\section{DISCLAIMER}

This report was prepared as an account of work sponsored by an agency of the United States Government. Neither the United States Government nor any agency Thereof, nor any of their employees, makes any warranty, express or implied, or assumes any legal liability or responsibility for the accuracy, completeness, or usefulness of any information, apparatus, product, or process disclosed, or represents that its use would not infringe privately owned rights. Reference herein to any specific commercial product, process, or service by trade name, trademark, manufacturer, or otherwise does not necessarily constitute or imply its endorsement, recommendation, or favoring by the United States Government or any agency thereof. The views and opinions of authors expressed herein do not necessarily state or reflect those of the United States Government or any agency thereof. 


\section{DISCLAIMER}

Portions of this document may be illegible in electronic image products. Images are produced from the best available original document. 


$$
\text { CONVERGENCE ANALYSIS OF }
$$

SOME FACTORIZATION ITERATIVE METHODS

FOR M-Matrices*

by

Robert Beauwens

\section{Abstract}

An improved definition and a new classification of factorization iterative methods are proposed. Factorizability conditions and convergence analysis are given for the class of M-matrices. Block factorization iterative methods are introduced and some essential properties of the point methods are generalized to block methods.

\footnotetext{
*Work supported by a NATO fellowship and in part by Brussels University, Northwestern University, and the U.S. Atomic Energy Commission under Contract No. AT (11-1)-2280(October 1973).
} 


\section{Introduction}

Factorization iterative methods have been introduced independently by Buleev [1] in 1959 and by Oliphant [2] [3] in 1960. They do not seem to have been used until the recent works of Stone [4], Dupont, Kendall and Rachford [5] and Dupont [6]. Stone introduced new methods; Dupont and his co-workers reformulated the method of Buleev and gave the first theoretical analysis.

Some of these methods have been rediscovered and new ones introduced by Woznicki in his thesis [7]. On the other hand, the work of Woznicki provides an original analysis based on the theory of regular splittings and a very useful matrix notation. It has initiated further developments [8]-[11]: a general approach, a review of the literature, a first classification and some basic lemma's were presented in [8] for the class of diagonally dominant matrices; general matrices were considered in [9]; intercomparison between factorization iterative methods were put in a general setting by means of an ordering concept in [10]; finally, the possible extension of these results to the class of M-matrices was indicated in [11].

In the present work, we attempt to improve the previous classification of the factorization iterative methods and we introduce block-factorization iterative methods. A general analysis is presented for the class of M-matrices. 


\section{Definitions and notations}

We shall investigate the solution of the linear system

$$
A x=b
$$

by iterative schemes of the form

$$
\mathrm{Mx}_{\mathrm{m}+1}=\mathrm{Nx} \mathrm{m}_{\mathrm{m}}+\mathrm{b}
$$

where $x_{m}$ denote the successive iterates and

$$
A=M-N
$$

We assume that $A$ is partitioned into sub-matricies $A_{i j}$, $i, j=1,2$.. n where the diagonal blocks $A_{i i}$ are square. Unless otherwise stated, we assume that all other matrices have the same order as $A$ and are partitioned in the same way.

When Eq. (3) is solved by means of a block triangular decomposition of $M$ in

$$
\mathrm{M}=\mathrm{IU}
$$

where $\mathrm{L}$ and $\mathrm{U}$ are respectively lower and upper block triangular matrices, the corresponding iterative method will be called a block factorization iterative method. When the point partitioning is used, the corresponding method will be called a point factorization iterative method.

We shall only consider iterative methods of this class; therefore, we assume that the decomposition (4) is feasible, i.e., that the diagonal blocks of $\mathrm{L}$ and $\mathrm{U}$ are non-singular.

It is clear that a particular method will be completely defined as soon as means are provided for computing the elements of $\mathrm{L}$ and $\mathrm{U}$; however, the decomposition (4) is not unique and it will be useful to put it in a standard form. Let $P_{I}$ and $P_{U}$ be the block-diagonal parts of $I$ and $U$ and define 


$$
\begin{aligned}
& L=P_{L}-E_{L} \\
& U=P_{U}-F_{U}
\end{aligned}
$$

so that $-E_{L}$ and $-F_{U}$ are respectively the strictly lower and upper block-triangular parts of $\mathrm{L}$ and $U$. We have then

$$
\begin{aligned}
M & =L U=\left(P_{L}-E_{L}\right)\left(P_{U}-F_{U}\right) \\
& =\left(P_{L} P_{U}-E_{L} P_{U}\right) P_{U}^{-1} P_{L}^{-1}\left(P_{L} P_{U}-P_{L} F_{U}\right)
\end{aligned}
$$

since $P_{L}$ and $P_{U}$ are assumed non-singular; thus

$$
M=\left(P-E_{1}\right) P^{-1}\left(P-F_{1}\right)
$$

where

$$
P=P_{I} P_{U} \quad E_{1}=E_{L} P_{U} \quad F_{1}=P_{L} F_{U}
$$

We shall henceforth take the decomposition (5) as the standard factorization of $M$; it has the advantage that, given $M$, the definition of $\mathrm{P}, \mathrm{E}_{1}$ and $\mathrm{F}_{1}$ is unique. Conversely, if $\mathrm{P}, \mathrm{E}_{1}$ and $\mathrm{F}_{1}$ are specified, the corresponding block factorization iterative method is completely defined. All particular classes of methods considered below will be defined in this way, by providing unique definitions for $P, E_{1}$ and $F_{1}$. Some standard notations, used in subsequent sections are summarized below.

We assume that

$$
A=D-E-F
$$

denotes the decomposition of the matrix A of Eq.(1) into its blockdiagonal, strictly lower and upper block-triangular parts respectively, according to the given partitioning.

The ordering $\mathrm{B} \leq \mathrm{C}$ of matrices or vectors of compatible dimensions is the usual component-wise ordering: $b_{i j} \leq c_{i j}$ for $a 11 i, j$. Similarly $B<C$ means $b_{i j}<c_{i j}$ for $a 11 i, j$. We say that $B$ is posive (non-negative) 
if $B>0(B \geq 0)$.

For diagonal matrices, we shall accept a slight deviation from these usual conventions: $P>0$ will mean that $p_{i i}>0$ for $i=1,2$. . $n$; there is no possibility of confusion since a diagonal matrix could hardly be said positive in any other sense.

If $R(B)$ is a matrix-valued function with the matrix $B$ as argument, we say that $R(B)$ is a non-decreasing (non-increasing) function of $B$ if $B \leq C$ implies $R(B) \leq R(C)(R(B) \geq R(C)$ ) and an increasing (decreasing) function of $B$ if $B \leq C$ equality excluded implies $R(B) \leq R(C)(R(B) \geq R(C))$ equality excluded. The same terminology will be used for matrix-valued functions of sets; ordered by the inclusion relation.

The spectral radius of a matrix $B$ will be denoted by $\rho(B)$. Given the matrix $B=\left(b_{i j}\right)$ and a set $W$ of couples of indices $(i, j)$ we denote by

$$
\operatorname{rest}_{W}(B)
$$

the matrix whose entries at $(i, j)$ are $b_{i j}$ if $(i, j) \in W$ and, otherwise are zero.

If $W$ denotes the set of diagonal (block-diagonal) indices, we use $\operatorname{diag}(B)$ (block-diag $(B))$ to denote $\operatorname{rest}_{W}(B)$; off-diag(B) denotes $B-d i a g(B)$ and block-offdiag(B) denotes B-block-diag(B).

It will also be useful to introduce a particular kind of matrix multiplication. Let $B=\left(b_{i j}\right)$ and $c=\left(c_{i j}\right)$ be two rectangular matrices of the same dimensions, we shall denote by $B \cdot C$ the matrix whose element $(i, j)$ is $b_{i j} c_{i j}$; we refer to this kind or product as the "element by element" product.

As a last remark, we want to warn the reader that, a1though we tried to avoid the use of the same symbol to denote different things, we did not however observe this rule strictly; the letter A for example is used as a "constant" to' denote the matrix of the given linear system (1) 
but also as a "variable" to denote any matrix as needed in statements like lemma's 1 to 6 . 
3. Classification of point factorization iterative methods

In this and the next section, we assume that the point partitioning is used; in other words, a11 blocks are scalars and all matrices are of order n.

\subsection{Basic methods}

31.1 The method of 01iphant

In 1962, Oliphant [3] introduced a family of iterative procedures which are defined, using the notations of section 2 by

$$
\begin{aligned}
& E_{1}=E \quad F_{1}=\beta F \\
& P=D \cdots \operatorname{diag}\left(\beta E P^{-1} F\right)
\end{aligned}
$$

where $\beta$ is a scalar parameter.

We shall use here a generalized definition, given by

$$
\begin{aligned}
& E_{1}=E_{B} \quad F_{1}=F_{\beta} \\
& P=D-\operatorname{diag}\left(E_{\beta} P^{-I_{F}}\right)
\end{aligned}
$$

where

$$
\begin{array}{ll}
E_{\beta}=-\left(\beta_{i s} a_{i s}\right) & (i, s) \in V_{E} \\
F_{\beta}=-\left(\beta_{s j} a_{s j}\right) & (s, j) \in V_{F}
\end{array}
$$

$a_{i j}$ are the entries of $A ; V_{E}$ and $V_{F}$ are the sets of couple of indices which correspond to non-zero entries of $E$ and $F$ respectively; $\beta_{i j}$ are arbitrary parameters defined for $(i, j) \in V=V_{E} U V_{F}$, the set of non-zero off-diagonal entries of $A$.

The corresponding splitting is given by

$$
\begin{aligned}
& M=\left(P-E_{\beta}\right) P^{-1}\left(P-F_{\beta}\right) \\
& N=\left(E-E_{\beta}\right)+\left(F-F_{\beta}\right)+\operatorname{off}-\operatorname{diag}\left(E_{\beta} P^{-1} F_{\beta}\right)
\end{aligned}
$$




\subsection{The method of Buleev}

Another family of factorization iterative methods was introduced by Buleev [1] in 1959 for solving the multidimensional diffusion equation. Similar methods have been used by Dupont, Kendal1 and Rachford [5† in 1968. As shown in $r 10 \mathrm{y}$, these families are equivalent; therefore we shall name them after their first author, i.e., Buleev. However, we shall use a definition which actually generalizes the notation of Dupont, Kenda11 and Rachford since it allows easier comparison with other methods.

Using the notation of section 2 , our definition may be stated as

$$
\begin{aligned}
& E_{1}=E \quad F_{1}=F \\
& P=(I+\alpha) D-\operatorname{diag}\left(E P^{-1} F S\right)
\end{aligned}
$$

where $\alpha$ is a diagonal matrix of arbitrary parameters, and $\mathrm{S}$ is the matrix whose entries are all equal to 1.

Alternatively, the definition of $P$ is given by the following recurrence relation

$$
P_{i i}=\left(1+\alpha_{i}\right) a_{i i}-\sum_{s=1}^{i-1} \sum_{j=s+1}^{n} \frac{a_{i s}{ }^{a} s j}{p_{s s}}
$$

where $P_{i i}, \alpha_{i}$ and $a_{i j}$ denote the entries of $P, \alpha$ and $A$, respectively.

The corresponding spitting is given by

$$
\begin{aligned}
& M=(P-E) P^{-1}(P-F) \\
& N=D_{\alpha}+\operatorname{off-diag}\left(E P^{-1} F\right)
\end{aligned}
$$

where $\mathrm{D}_{\alpha}$ is a diagonal matrix with entries

$$
\left(D_{\alpha}\right)_{i i}=\alpha_{i} a_{i i}-\sum_{s=1}^{i-1} \sum_{\substack{j=s+1 \\ j \neq i}}^{n} \frac{a_{i s}{ }^{a} s j}{p_{s s}}
$$

An essential characteristic of the method of Buleev is the appearance of a non-zero main diagonal in the matrix $N$. For the other basic methods, the matrix is purely off-diagonal. 
Related methods have been considered by Stone [4]; however, we shall not consider them here since no rigorous analysis has been obtained for these procedures so far.

\subsection{The method of Woznicki}

In 1973, Woznicki [7] introduced another basic family of "two-sweep" iterative methods defined, in the notation of section 2, by

$$
\begin{array}{rr}
E_{1}=E+G \quad F_{1}=F+G \\
P=D-\operatorname{diag}(E+G) P^{-1}(F+H) \\
G+H=\operatorname{rest}_{W}\left((E+G) P^{-1}(F+H)\right)
\end{array}
$$

where $G$ and $H$ are respectively strictly lower and upper triangular matrices and $W$ is an arbitrary sei of off-diagonal entries.

It is seen that $P, G$, and $H$ are defined by implicit equations which allow the recursive computation of their elements, provided $P$ is not singular; equations (22) and (23) are indeed equivalent to

$$
\begin{aligned}
& p_{i i}=a_{i i}-\sum_{s=1}^{i-1\left(a_{i s}-g_{i s}\right)\left(a_{s i}-h_{s i}\right)} p_{s s} \\
& g_{k i}= \begin{cases}\sum_{s=1}^{i-1\left(a_{k s}-g_{k s}\right)\left(a_{s i}-h_{s i}\right)} & \text { if }(k, i) \in W \\
0 & \text { if }(k, i) \notin W\end{cases} \\
& h_{i k}=\left\{\begin{array}{lll}
i-1 \frac{\left(a_{i s}-g_{i s}\right)\left(a_{s k}-h_{s k}\right)}{p_{s s}} & \text { if }(i, k) \in W \\
0 & \text { if }(i, k) \notin W
\end{array}\right.
\end{aligned}
$$

with

$$
p_{11}=a_{11} \quad g_{k 1}=h_{1 k}=0 \quad k=2,3 \ldots . n
$$

where $p_{i i}, a_{i j}, g_{i s}$ and $h_{s i}$ denote the elements of $P, A, G$ and $H$ respectively. The elements of $P, G$ and $H$ are determined in $n$ steps; during the ith step, 
$\mathrm{p}_{i \mathrm{i}}, \mathrm{g}_{\mathrm{ki}}$ and $\mathrm{h}_{\mathrm{ik}}$ are computed for $\mathrm{k}=\mathrm{i}+1, \mathrm{i}+2, \ldots, \mathrm{n}$.

It should be observed that some entries of $G+H$, with $(i, j) \in W$

may be zero; for example, as pointed out by Woznicki $\Gamma 71$, the elements of the flrst row or of the first column are always identically zero. We shall later need a more restrictive definition of $\mathrm{W}$; therefore; we assume henceforth that such entries are deleted from the set $W$. We denote by $W_{G}$ and $W_{H}$ the sets of non-zero entries of $G$ and $H$ respectively.

The splitting which corresponds to the method of Woznicki is given by

$$
\begin{aligned}
& M=(P-E-G) P^{-1}(P-F-H) \\
& N=\operatorname{off-diag}\left((E+G) P^{-1}(F+H)\right)-(G+H)
\end{aligned}
$$

It is worthwhile to observe that the Choleski factorization is a particular case of this family; in this case, however, the method ceases to be iterative since $\mathrm{N}$ is zero.

\subsection{Composite methods}

In the basic families considered so far, one method occupies a central position because it belongs to all families. This method, called EWA by Woznicki $\Gamma 77$ is defined by

$$
\begin{aligned}
& E_{1}=E \quad F_{1}=F \\
& P=D-\operatorname{diag}\left(E P^{-1} F\right)
\end{aligned}
$$

It is obtained from the 0liphant method by. setting all the parameters $\beta_{i j}$ equal to 1 , from the Buleev method by using $\alpha_{1}=0$ and

$$
\alpha_{i}=\frac{1}{a_{i i}} \sum_{s=1}^{i-1} \sum_{\substack{j=s+1 \\ j \neq s}}^{n} \frac{a_{i s} a_{s j}}{p_{s s}}
$$

for $i>1$ in conjunction with the recurrence (18), and from the method of Woznicki by setting $G$ and $H$ to zero.

We may thus consider the methods of 0liphant, Buleev, and Woznicki. 
as three basic generalizations of this particular method. By combining these families together, we can generate composite procedures which generalize and unify these basic schemes.

We briefly summarize below the definitions of the composite methods, using obvious notations.

32.1 The Oliphant-Buleev method (OB)

$$
\begin{aligned}
& E_{1}=E_{\beta} \quad F_{1}=F_{\beta} \\
& P=(I+\alpha) D-\operatorname{diag}\left(E_{\beta} P^{-1} F_{\beta} S\right) \\
& E_{\beta}=-\left(\beta_{i s} a_{i s}\right) \quad(i, s) \in V_{E} \\
& F_{\beta}=-\left(\beta_{s j}^{a} s j\right) \quad(s, j) \in V_{F} \\
& M=\left(P-E_{\beta}\right) P^{-1}\left(P-F_{\beta}\right) \\
& N=D_{\alpha}+\left(E-E_{\beta}\right)+\left(E-F_{\beta}\right)+\operatorname{off}-\operatorname{diag}\left(E_{\beta} P^{-1} F_{\beta}\right) \\
& \left(D_{\alpha}\right)_{i i}=\alpha_{i} a_{i i}-\sum_{s=1}^{i-1} \sum_{\substack{j=s+1 \\
j \neq i}}^{n} \frac{\beta_{i s_{i s}{ }^{a} s j^{a} j}}{p_{s s}}
\end{aligned}
$$

32.2 The oliphant-Woznicki method (OW)

$$
\begin{aligned}
E_{1} & =E_{\beta}+G \quad F_{1}=F_{\beta}+H \\
P & =D-\operatorname{diag}\left(\left(E_{\beta}+G\right) P^{-1}\left(F_{\beta}+H\right)\right) \\
G+H & =r e s t_{W}\left(\left(E_{\beta}+G\right) P^{-1}\left(F_{\beta}+H\right)\right) \\
E_{B} & =-\left(\beta_{i s} a_{i s}\right) \\
F_{B} & =-\left(\beta_{s j} a_{s j}\right) \\
M & =\left(P-E_{\beta}-G\right) P^{-1}\left(P-F_{\beta}-H\right) \\
N & \left(E-E_{E}\right.
\end{aligned}
$$


32.3 The Buleev-Woznicki method (BW)

$$
\begin{aligned}
E_{1} & =E+G \quad F_{1}=F+H \\
P & =(I+\alpha) D-\operatorname{diag}\left((E+G) P^{-1}(F+H) S\right) \\
G+H & =\operatorname{rest}_{W}\left((E+G) P^{-1}(F+H)\right) \\
M & =(P-E-G) P^{-1}(P-F-H) \\
N & =D_{\alpha}+\left[\operatorname{off}-\operatorname{diag}\left((E+G) P^{-1}(F+H)\right)-(G+H)\right] \\
\left(D_{\alpha}\right)_{i i} & =\alpha_{i} a_{i i}-\sum_{s=1}^{i-1} \sum_{j=s+1}^{n} \frac{\left(a_{i s}-g_{i s}\right)\left(a_{s i}-h_{s i}\right)}{P_{s s}}
\end{aligned}
$$

32.4 The Oliphant-Buleev-Woznicki method (OBW)

$$
\begin{aligned}
& E_{1}=E_{\beta}+G \quad F_{1}=F_{\beta}+H \\
& P=(I+\alpha) D-\operatorname{diag}\left(\left(E_{\beta}+G\right) P^{-1}\left(F_{\beta}+H\right) S\right) \\
& G+H=\operatorname{rest}_{W}\left(\left(E_{\beta}+G\right) P^{-1}\left(F_{\beta}+H\right)\right) \\
& E_{\beta}=\infty\left(\beta_{\text {is.is }}\right) \quad(i, s) \in W_{E} \\
& F_{\beta}=-\left(\beta_{s j} a_{s j}\right) \quad(s, j) \in W_{F} \\
& M=\left(P-E_{B}-G\right) P^{-1}\left(P-F_{B}-H\right) \\
& N=D_{\alpha}+\left(E-E_{\beta}\right)+\left(F-F_{\beta}\right)+\left[\operatorname{off}-\operatorname{diag}\left(\left(E_{\beta}+G\right) P^{-1}\left(F_{\beta}+H\right)\right)-(G+H)\right] \\
& \left(D_{\alpha}\right)_{i i}=\alpha_{i} a_{i i}-\sum_{s=1}^{i-1} \sum_{\substack{j=s+1 \\
j \neq i}}^{n} \frac{\left(\beta_{i s} a_{i s}-g_{i s}\right)\left(\beta_{s j}{ }^{a} s_{j}-h_{s j}\right)}{p_{s s}}
\end{aligned}
$$


4. Factorization conditions and convergence analysis of point methods for M-matrices

4.1 M-matrices and regular splittings

We sumnarize briefly the properties of M-matrices and regular

splittings which will be used in subsequent sections.

Definition 1 (Co1latz「15])

A real $n \times n$ matrix $A$ is monotone if $A x \geq 0$ implies $x \geq 0$.

It is equivalent to state that $A$ is non-singular and $A^{-1} \geq 0$.

Definition 2 (Varga $[12\rceil$ )

A real $n \times n$ matrix $A=\left(a_{i j}\right)$ with $a_{i j} \leq 0$ for $i \neq j$ is an Mmmatrix if $\mathrm{A}$ is monotone.

For the next theorem and related properties, we refer to the works of Ky Fan [13] and Fiedler and Pták [14].

Theorem 1 (Ky Fan [137)

Let $A=\left(a_{i j}\right)$ be a real $n \times n$ matrix such that $a_{i j} \leq 0$ for $i \neq j$. Then $A$ is monotone if and only if there exists $x \geq 0$ such that $A x>0$.

As a consequence, it may be observed that if $A=\left(a_{i j}\right)$ and $A^{\prime}=\left(a^{\prime}{ }_{i j}\right)$ with $a_{i j}$ and $a_{i j} \leq 0$ for $i \neq j$ are such that $A$ is an M-matrix and $A^{\prime} \geq A$, then $A^{*}$ is also an M-matrix and $A^{-1} \geq A^{-1}$.

In particular if $\mathrm{A}$ is a partitioned M-matrix, its block diagonal D is an M-matrix and therefore each block of $D$ is an M-matrix; moreover $A^{-1} \geq D^{-1}$.

Similarly $\operatorname{diag}(A)=\left(a_{i i}\right)$ is an M-matrix, thus positive and $A^{-1} \geq\left(a_{i i}^{-1}\right)$.

Definition 2 (varga $[12]$ )

A splitting $A=M-N$ is regular when $M$ is non-singular with $M^{-1} \geq 0$ and $\mathrm{N} \geq 0$.

Moreover we shall say that a splitting is convergent when the corresponding iterative method is convergent. 
The following theorem, due to Woznicki [7] is a sharpening of theorem 3.15 of Varga [12].

Theorem 2 (Woznicki [7]̣).

Let $A=M_{1}-N_{1}=M_{2}-N_{2}$ be two regular splittings of $A$ where $A^{-1} \geq 0$. If $M_{1}^{-1} \geq M_{2}^{-1} \geq 0$ equality excluded, then

$$
\rho\left(M_{1}^{-1} N_{1}\right) \leq \rho\left(M_{2}^{-1} N_{2}\right)<1
$$

the inequality being strict when $A^{-1}>0$.

\subsection{The 0liphant method}

A factorization algorithm fails if $\mathrm{P}$ is singular. Conditions which insure that $P$ is not singular (actually positive) will be investigated; as a consequence, convergence rate comparisons will be obtained.

\section{Lemma 1}

Let $\mathrm{A}=\mathrm{K}-\mathrm{L}-\mathrm{U}$ where $\mathrm{K} \geq 0, \mathrm{~L} \geq 0$, and $\mathrm{U} \geq 0$ are respectively diagona1, strictly lower and upper triangular $\mathrm{n} \times \mathrm{n}$ matrices, and let the diagonal matrix $P$ be defined by

$$
P=K-\operatorname{diag}\left(L P^{-1} U\right)
$$

If $\mathrm{A}$ is an M-matrix, $\mathrm{P}$ is positive; moreover $\mathrm{P}$ is a non-decreasing function of A.

Proof

Let $\mathrm{k}_{S S}, \mathrm{p}_{S \mathrm{~S}}, \mathrm{l}_{\mathrm{is}}$, and $\mathrm{u}_{\mathrm{si}}$ denote the elements of $\mathrm{K}, \mathrm{P}, \mathrm{I}$ and $\mathrm{U}$ respectively. Since $A$ is monotone, there exists $x \geq 0$ such that $A x>0$. We prove by induction that the sth component of $(P-U) x^{\prime}$ is also positive, which in turn implies $\mathrm{p}_{\mathrm{SS}}>0$.

The proposition is true for $s=1$ since $p_{11}=k_{11}$ and $((P-U) x)_{1}=(A x)_{1}>0$. For $s>1$ we have

$$
((P-U) x)_{s}=\left(k_{s s}-\sum_{t=1}^{s-1} \frac{1 s u^{u} t s}{p_{t t}}\right) x_{s}-\sum_{j=s+1}^{n} u_{s j} x_{j}
$$


But for $t \leq s-1$,

$$
P_{t t} x_{t}>\sum_{j=t+1}^{n} u_{t j} x_{j} \geq u_{t s} x_{s}
$$

by the induction hypothesis; thus $\mathrm{P}_{t t}>0$ and

$$
x_{t}>\frac{u_{t s}}{p_{t t}} x_{s}
$$

Therefore

$$
((P-U) x)_{s} \geq k_{s s} x_{s}-\sum_{t=1}^{s-1} 1_{s t} x_{t}-\sum_{j=s+1}^{n} u_{s j} x_{j}=(A x)_{s}>0 .
$$

and $\mathrm{p}_{\mathrm{sS}}>0$

For the second part of the lemma, assume with obvious notations that $\dot{A}^{\prime}=\dot{K}^{\prime}-L^{\prime}-U^{\prime}$ with $K^{\prime} \geq 0, L^{\prime} \geq 0$ and $U^{\prime} \geq 0$ is such that $A^{\prime} \geq A$. Since these conditions imply that $A^{\prime}$ is an M-matrix, $P^{\prime}$ is positive by applying the first part of the lemma.

On the other hand we have

$$
\mathrm{p}_{11}^{\prime}=\mathrm{k}_{11}^{\prime} \geq \mathrm{k}_{11}=\mathrm{p}_{11}
$$

and, assuming $\mathrm{P}_{t t}^{\prime} \geq \mathrm{p}_{t t}$ for $t \leq s-1$, we have

$$
p_{s s}^{\prime}=k_{s s}^{\prime}-\sum_{t=1}^{s-1} \frac{1_{s t}^{\prime} u_{s t}^{\prime}}{p_{t t}^{\prime}} \geq k_{s s}-\sum_{t=1}^{s-1} \frac{1 s^{u} t s}{p_{t t}}=p_{s s}
$$

An immediate consequence is

\section{Coro11ary}

If $A$ is an M-matrix, the diagonal matrix $P$ of the oliphant method is positive when $0 \leq \beta_{i j} \leq 1$ for $(i, j) \in V$. Moreover $P$ is a non-increasing function of $\beta_{i j}$ for $0 \leq \beta_{i j} \leq 1,(i, j) \in V$.

\section{Theorem 3}

If $A$ is an M-matrix and $0 \leq \beta_{i j} \leq 1$ for $(i, j) \in V$, then the oliphant method is convergent.

If $T=M^{-1} N$ is the associated iterative matrix, $\rho(T)$ is a non-increasing function of $\beta_{i j}$ for $0 \leq \beta_{i j} \leq 1(i, j) \in V$. If moreover $A^{-1}>0, \rho(T)$ is a 
decreasing function of $\beta_{i j}$ for $0 \leq \beta_{i j} \leq 1,(i, j) \in V$.

Proof

From section 3, Eq. (14) and (15) we have

$$
\begin{aligned}
& M=P\left(I-P^{-1} E_{\beta}\right)\left(I-P^{-1} F_{\beta}\right) \\
& N=\left(E-E_{\beta}\right)+\left(F-F_{\beta}\right)+\operatorname{off}-\operatorname{diag}\left(E_{\beta} P^{-1} F_{\beta}\right)
\end{aligned}
$$

The first two terms of $N$ are obviously non-negative for $0 \leq \beta_{i j} \leq 1$.

The third term is non-negative since $\mathrm{P}>0$ by lemma 1 . Thus $\mathrm{N} \geq 0$.

On the other hand we have

$$
M^{-1}=\left(I-P^{-1} F_{\beta}\right)^{-1}\left(I-P^{-1} E_{\beta}\right)^{-1} P^{-1}
$$

and $\mathrm{P}>0$; using the expansions

$$
\begin{aligned}
& \left(I-P^{-1} F_{\beta}\right)^{-1}=\sum_{k=0}^{n-1}\left(P^{-1} F_{\beta}\right)^{k} \\
& \left(I-P^{-1} E_{\beta}\right)^{-1}=\sum_{k=0}^{n-1}\left(P^{-1} E_{\beta}\right)^{k}
\end{aligned}
$$

it is seen that $\left(I-P^{-1} F_{B}\right)^{-1} \geq 0$ and $\left(I-P^{-1} E_{\beta}\right) \geq 0$. Therefore $M^{-1} \geq 0$. Thus the splitting $A=M-N$ is a regular splitting and, since $A$ is an M-matrix, it is convergent.

To prove the second, part of the theorem, assume that

$$
\beta_{i j} \leq \beta_{i j}^{\prime} \text { for all }(i, j) \in V \text {, equality excluded }
$$

(i.e., with strict inequality for at least one entry $(i, j)$ ), which implies

$$
\begin{aligned}
& E_{\beta}, \geq E_{\beta} \\
& F_{\beta}, \geq F_{\beta}
\end{aligned}
$$

where equality is obviously excluded for at least one of these inequalities. 
By lemma 1 we have

$$
\mathrm{P}^{-1} \geq \mathrm{P}^{-1}>0
$$

and, using expansions (71), (72),

$$
M^{-1} \geq M^{-1} \text { equality excluded }
$$

The conclusion follows, by applying theorem 2 .

\subsection{The Oliphant-Buleev method}

Lemma 2

Let $A=K-L-U$ where $K \geq 0, L \geq 0$, and $U \succeq 0$ are respectively diagonal, strictly lower and upper triangular matrices, and let $\alpha$ be a diagonal matrix.

Let $P$ be the diagonal matrix whose entries $p_{i i}$ are defined by the recurrence formula

$$
p_{i i}=\left(1+\alpha_{i}\right) k_{i i}-\sum_{s=1}^{i-1} \sum_{j=s+1}^{n} \frac{1_{i s} u_{s j}}{p_{s s}}
$$

where $\alpha_{i}, k_{i i}, 1_{i s}$, and $u_{s j}$ are the entries of $\alpha, k, L$, and $U$ respectively. Let $\alpha^{\circ}$ and $\mathrm{P}^{\circ}$ be defined by the recurrence relations

$$
\begin{aligned}
& p_{i i}^{o}=\left(1+\alpha_{i}^{o}\right) k_{i i}-\sum_{s=1}^{i-1} \sum_{j=s+1}^{n} \frac{1 s^{u} s i}{p_{s s}^{o}} \\
& \alpha_{i}^{o}=\frac{1}{k_{s s}} \sum_{s=1}^{i-1} \sum_{j=s+1}^{n} \frac{1 s_{s j}^{u}}{p_{s s}^{o}} \text { with } \alpha_{1}^{o}=0
\end{aligned}
$$

Then, if $\mathrm{A}$ is an M-matrix and $\alpha \geq \alpha^{\circ}, \mathrm{P}$ is positive; moreover $\mathrm{P}$ is a non-decreasing function of $A$ and an increasing function of $\alpha$ in this range. 
$\underline{\text { Proof }}$

The last part of the lemma is easily proven by induction under the assumption that $\mathrm{P}>0$. But for $\alpha=\alpha^{0}, \mathrm{P}$ is positive by lemma 1. The proposition follows.

As an immediate consequence, we have:

Coro11ary

If $A$ is an M-matrix, $0 \leq \beta_{i j} \leq 1$ for $(i, j) \in V$ and $\alpha \geq \alpha^{\circ}$, then the diagonal matrix $P$ of the Oliphant-Buleev method is positive. Moreover $P$ is a non-decreasing function of $\beta_{i j}$ for $0 \leq \beta_{i j} \leq 1,(i, j) \in V$ and an increasing function of $\alpha$ for $\alpha \geq \alpha^{\circ}$.

It should be observed that $\alpha^{0}$ is not a constant for the 0liphantBuleev method since it depends on the parameters $\beta_{i j}$. Actually $\alpha^{\circ}$ is the value of $\alpha$ which reduces the 0liphant-Buleev method to the 01iphant method.

Theorem 4

If $A$ is an M-matrix, $0 \leq \beta_{i j} \leq 1$ for $(i, j) \in V$ and $\alpha \geq \alpha^{\circ}$, then the oliphant-Buleev method is convergent.

If $T=M^{-1} N$ is the associated iteration matrix, $\rho(T)$ is a decreasing function of $\beta_{i j}$ for $0 \leq \beta_{i j} \leq 1,(i, j) \in V$ and an increasing function of $\alpha \geq \alpha^{\circ}$.

$\underline{\text { Proof }}$

From section 3, Eq. (36) - (38), we have

$$
\begin{aligned}
M & =P\left(I-P^{-1} E_{\beta}\right)\left(I-P^{-1} F_{\beta}\right) \\
N & =D_{\alpha}+\left(E-E_{\beta}\right)+\left(F-F_{\beta}\right)+\operatorname{off-diag}\left(E_{\beta} P^{-1} F_{\beta}\right) \\
\left(D_{\alpha}\right)_{i i} & =\alpha_{i} d_{i i}-\sum_{s=1}^{i-1} \sum_{\substack{j=s+1 \\
j \neq i}}^{n} \frac{\beta_{i s_{i s}} e_{s j} E_{s j}}{P_{s s}}
\end{aligned}
$$


From lemma 2, it is seen that $\left(D_{\alpha}\right)_{i i} \geq 0$ and $P^{-1}>0$ for

$0 \leq \beta_{i j} \leq 1$ and $\alpha \geq \alpha^{\circ}$; the other terms of $N$ are obvious $1 y$ non-negative for $0 \leq \beta_{i j} \leq 1$. Thus $N \geq 0$.

On the other hand, the proof of theorem 3 applies to show that $M^{-1} \sum 0$. Thus, the splitting is regular and, since $A$ is an $M-$ matrix, it is convergent.

Similarly, the proof of theorem 3 is used to show that $\beta_{i j} \leq \beta_{i j}$ for all $(i, j) \in V$, equality excluded implies $M^{-1} \geq M^{-1}$ equality expluded. Moreover

$$
\alpha_{i}^{\prime} \leq \alpha_{i} \quad i \in[1, n] \text { equality excluded }
$$

implies, by lemma 2

$$
P^{-1} \geq P^{-1} \text { equality excluded }
$$

thus, by using expansions (71) and (72) for the factors of $M^{-1}$ and $M-1$,

$$
M^{-1} \geq M^{-1} \text { equality excluded }
$$

and the conclusion follows by applying theorem 2 .

\section{Remarks}

Using the proof of lemma 3 of ref [8], it is easily seen that the results of lemma 2 and its corollary can be extended to $\alpha>0$ for diagonally dominant M-matrices and to $\alpha \geq 0$ for strictly or irreducibly diagonally dominant M-matrices. However this extension is not possible for theorem 4 since for $\alpha \leq \alpha^{\circ}$ equality excluded, some diagonal entries of $\mathrm{N}$ become negative and the splitting is no longer regular. 


\subsection{The Oliphant-Woznicki method}

Lerma 3

Let $A=R-L-U$ where $K \geq 0, L \geq 0$ and $U \geq 0$ are respectively diagonal, strictly lower and upper triangular matrices, and let the diagonal, strictly lower and upper triangular matrices $P, G$ and $H$ respectively be defined by

$$
\begin{aligned}
P & =K-\operatorname{diag}\left((L+G) P^{-1}(U+H)\right) \\
G+H & =\operatorname{rest}_{W}\left((L+G) P^{-1}(U+H)\right)
\end{aligned}
$$

where $W$ is a given set of off-diagonal entries (deleting zero entries).

If $A$ is an M-matrix, $P$ is positive, $G$ and $H$ are non-negative; moreover $P$ is a non-decreasing function of $A$ and a non-increasing function of $W$; $G$ and $H$ are non-increasing functions of $A$ and $G+H$ is an increasing function of $W$.

\section{Proof}

We first prove the last parts of the lemma under the assumption that $P$ is positive if $A$ is an M-matrix.Letting $p_{i j}, k_{i i}, 1_{i s}, g_{i s}, u_{s j}$ and $h_{s j}$ denote the entries of the matrices $P, K, L, G, U$ and $H$ respectively, the relation defining $P, G$ and $H$ may be written

$$
\begin{aligned}
& p_{i i}=k_{i i}-\sum_{s=1}^{i-1} \frac{\left(1_{i s}+g_{i s}\right)\left(u_{s i}+h_{s i}\right)}{p_{s s}} \\
& g_{k i}= \begin{cases}\sum_{s=1}^{i-1} \frac{\left(I_{k s}+g_{k s}\right)\left(u_{s i}+h_{s i}\right)}{p_{s s}} & \text { if }(k, i) \in W \\
0 & \text { if }(k, i) \notin W\end{cases}
\end{aligned}
$$




$$
h_{i k}=\left\{\begin{array}{lll}
i-s & \frac{\left(1_{i s}+g_{i s}\right)\left(u_{s k}+h_{s k}\right)}{p_{s s}} & \text { if }(i, k) \in W \\
0 & \text { if }(i, k) \notin W & k>i
\end{array}\right.
$$

On the other hand, consider, with obvious notations that $A^{\prime}=K^{*}-L^{\prime}-U^{*}$ with $K^{\prime} \geq 0, L^{\prime} \geq 0$ and $U^{+} \geq 0$ is such that $A^{+} \geq A$. By formula's (89), (90) and (91), it is seen that

$$
\begin{aligned}
& \mathrm{p}_{11}^{\prime}=\mathrm{k}_{11}^{\prime} \geq \mathrm{k}_{11}=\mathrm{p}_{11} \\
& \mathrm{~g}_{\mathrm{k} 1}^{\circ}=\mathrm{g}_{\mathrm{k}: 1}=\mathrm{h}_{1 \mathrm{k}}=\mathrm{h}_{1 \mathrm{k}}^{\prime}=0 \text { for } \mathrm{k}>1
\end{aligned}
$$

and that

$$
\begin{array}{ll}
\mathrm{g}_{\mathrm{ks}}^{\circ} \leq \mathrm{g}_{\mathrm{ks}} & \mathrm{k}>\mathrm{s} \\
\mathrm{h}_{\mathrm{sk}}^{\mathrm{*}} \leq \mathrm{h}_{\mathrm{sk}} & \mathrm{k}>\mathrm{s}
\end{array}
$$

for $s \leq i-1$ together with $A^{\prime} \geq A$ imply

$$
\begin{aligned}
& \mathrm{g}_{\mathrm{ki}}{ }^{\mathrm{N}} \leq \mathrm{g}_{\mathrm{ki}} \quad \mathrm{k}>\mathrm{i} \\
& \mathrm{h}_{\mathrm{ik}} \mathrm{h}_{\mathrm{ik}} \quad \mathrm{k}>\mathrm{i} \\
& p_{i i}^{\prime} \geq p_{i i}
\end{aligned}
$$

Therefore $P$ is a non-decreasing function of $A$ and $G$ and $H$ are nonincreasing functions of $A$ under the assumptions that $P$ is positive and $A$ has non-positive off-diagonal elements.

$$
\begin{aligned}
& \text { Similarly, if } \mathrm{W} \text { is decreased to } \mathrm{W}^{\prime} \text {, we have } \\
& \qquad \mathrm{p}_{11}^{\prime}=\mathrm{p}_{11}=\mathrm{k}_{11} \\
& \qquad \mathrm{~g}_{\mathrm{k} 1}^{\prime}=\mathrm{g}_{\mathrm{k} 1}=\mathrm{h}_{1_{k}}=\mathrm{h}_{1_{k}}^{\prime}=0, \mathrm{k}>1
\end{aligned}
$$

and the relation

$$
\mathrm{g}_{\mathrm{ks}}^{\prime} \leq \mathrm{g}_{\mathrm{ks}}
$$




$$
\begin{aligned}
& h_{k s}^{\prime} \leq h_{s k}^{\prime} \\
& p_{s s}^{\prime} \geq p_{s s} .
\end{aligned}
$$

for $s \leq i-1$ together with $P^{\prime}>0$,imply

$$
\begin{aligned}
& g_{k i}^{\prime} \leq g_{k i} \\
& h_{i k}^{\prime} \leq h_{i k} \\
& p_{i i}^{\prime} \geq p_{i i}
\end{aligned}
$$

since each term of (90) and (91) may only be decreased and the same is true for the absolute value of each negative term of (89) while the positive one is not modified.

Moreover, if equality is excluded between $W$ and $W^{\prime}$ then equality is excluded between $G+H$ and $G^{\prime}+H^{\prime}$ since zero entries are exluded from $W$ and $W^{\prime}$.

Therefore $P$ is a non-increasing function of $W$ while $G+H$ is an increasing function of $W$ under the assumption that $P$ is positive.

Thus, it is sufficient to prove that $P$ is positive under the assumptions of the lemma when $\mathrm{W}$ is maximal and, for this purpose, we may obviously assume that $\mathrm{W}$ is the complete set of off-diagonal entries; the relations defining $P, G$ and $H$ may then be written

$$
\begin{array}{ll}
p_{i i}=k_{i i}-\sum_{s=1}^{i-1} \frac{\left(1_{i s}+g_{i s}\right)\left(u_{s i}+h_{s i}\right)}{p_{s s}} & \\
g_{k i}=\sum_{s=1}^{i-1} \frac{\left(1_{k s}+g_{k s}\right)\left(u_{s i}+h_{s i}\right)}{p_{s s}} & k>i \\
h_{i k}=\sum_{s=1}^{i-1} \frac{\left(1_{i s}+g_{i s}\right)\left(u_{s k}+h_{s k}\right)}{p_{s s}} & k>i
\end{array}
$$

Since $A$ is monotone, there exists $x \geq 0$ such that $A x>0$. We prove by induction the following set of relations 


$$
\begin{array}{ll}
g_{t s} \geq 0 \quad & \text { for } t>s \\
h_{s t} \geq 0 \quad & \text { for } t>s \\
((P-U-H) x)_{s}>0 & \\
p_{s s}>0 &
\end{array}
$$

These relations are satisfied for $s=1$ since we have

$$
\begin{aligned}
& g_{t 1}=h_{1 t}=0 \\
& ((P-U-H) x)_{1}=(A x)_{1}>0 \\
& p_{11}=k_{11}>0
\end{aligned}
$$

Assume that they hold for $s<i$; then

$$
g_{k i} \geq 0 \text { and } h_{i k} \geq 0 \text { for } k>1
$$

from equations (108), (109) since $\mathrm{p}_{\mathrm{sS}}>0$.

On the other hand, we have, from (107) and (109),

$$
\begin{aligned}
\left((P-U-H)_{x}\right)_{i} & =\left(k_{i i}-\sum_{s=1}^{i-1} \frac{\left(1_{i s}+g_{i s}\right)\left(u_{s i}+h_{s i}\right)}{p_{s s}}\right) x_{i}-\sum_{k=i+1}^{n}\left(u_{i k}+h_{i k}\right) x_{k} \\
& =k_{i i} x_{i}-\sum_{s=1}^{i-1} \sum_{k=i}^{n} \frac{\left(1_{i s}+g_{i s}\right)\left(u_{s k}+h_{s k}\right)}{p_{s s}} x_{k}-\sum_{k=i+1}^{n} u_{i k} x_{k}
\end{aligned}
$$

Defining the sequence of parameters $\alpha_{s}^{p}$ by the recursive relation

$$
\alpha_{s}^{p}=\sum_{k=s+1}^{i-p} \frac{u_{s k}+h_{s k}}{p_{s s}} \alpha_{k}^{(p-1)}
$$

for $\mathrm{p}>0$, with

$$
\alpha_{s}^{0}=\sum_{k=i}^{n} \frac{u_{s k}{ }^{+h} s k}{p_{s s}} x_{k}
$$

it is seen that 


$$
\begin{aligned}
\sum_{s=1}^{i-p} g_{i s} \alpha_{s}^{(p-1)} & =\sum_{s=1}^{i-p} \sum_{k=1}^{s-1} \frac{\left(1_{i k}+g_{i k}\right)\left(u_{k s}+h_{k s}\right)}{p_{k k}} \alpha_{s}^{(p-1)} \\
& =\sum_{k=1}^{i-p-1} \sum_{s=k+1}^{i-p} \frac{\left(1_{i k}+g_{i k}\right)\left(u_{k s}+h_{k s}\right)}{p_{k k}} \alpha_{s}^{(p-1)} \\
& =\sum_{k=1}^{i \infty p-1}\left(1_{i k}+g_{i k}\right) \alpha_{k}^{p}=\sum_{s=1}^{i-p-1}\left(1_{i s}+g_{i s}\right) \alpha_{s}^{p}
\end{aligned}
$$

and, by repeated use of this relation,

$$
\sum_{s=1}^{i-1}\left(1_{i s}+g_{i s}\right) \alpha_{s}^{o}=\sum_{s=1}^{i-1} \sum_{p=0}^{i-s-1} 1_{i s} \alpha_{s}^{p}
$$

On the other hand, we have $\alpha_{s}^{0}<x_{s}$ by the induction hypothesis since $s \leq i-1$. Then, assuming

$$
\sum_{p=0}^{q-1} \alpha_{s}^{p}<x_{s}
$$

we have

$\sum_{p=0}^{q} \alpha_{s}^{p}=\sum_{k=1}^{n} \frac{u_{s k}+h_{s k}}{p_{s s}} x_{k}+\sum_{p=0}^{q-1} \sum_{k=s+1}^{i-p-1} \frac{u_{s k}+h_{s k}}{p_{s s}} \alpha_{k}^{p}<\sum_{k=s+1}^{n} \frac{u_{s k}+h_{s k}}{p_{s s}} x_{k}<x_{s}$

which proves that

$$
\sum_{p=0}^{q} \alpha_{s}^{p}<x_{s}
$$

holds for all values of $q$.

Then

$$
\begin{aligned}
((P-U-H) x)_{i} & =k_{i i} x_{i}-\sum_{s=1}^{i-1}\left(1_{i s}+g_{i s}\right) \alpha_{s}^{0}-\sum_{k=i+1}^{n} u_{i k} x_{k} \\
& =k_{i i} x_{i}-\sum_{s=1}^{i-1} 1_{i s}\left(\sum_{p=0}^{i-s-1} \alpha_{s}\right)-\sum_{k=i+1}^{n} u_{i k} x_{k} \\
& =k_{i i} x_{i}-\sum_{s=1}^{i-1} 1_{i s} x_{s}-\sum_{k=i+1}^{n} u_{i k} x_{k}=(A x)_{i}>0
\end{aligned}
$$


and this entails

$$
p_{\text {ii }}>0
$$

\section{Corollary}

If $A$ is an M-matrix and $0 \leq \beta_{i j} \leq 1$ for $(i, j) \in V$, then the diagonal matrix $P$ of the Oliphant-Woznicki method is positive while the off-diagonal matrix $G+H$ is nonnegative. Moreover $P$ is a non-increasing function of $\beta_{i j}$ for $0 \leq \beta_{i j} \leq 1,(j, j) \in V$ and of $W ; G$ and $H$ are non-decreasing functions of $\beta_{i j}$ for $0 \leq \beta_{i j} \leq 1,(i, j) \in V$ and $G+H$ is an increasing function of $W$.

\section{Theorem 5}

If $A$ is an M-matrix and $0 \leq \beta_{i j} \leq 1$ for $(i, j) \in V$, then the oliphantWoznicki method is convergent.

If $T=M^{-1} N$ is the associated iteration matrix, $\rho(T)$ is a nonincreasing function of $\beta_{i j}$ for $0 \leq \beta_{i j} \leq 1,(i, j) \in V$ and of $W_{0}$

If, moreover, $A^{-1}>0, \rho(T)$ is a decreasing function of $\beta_{i j}$ for $0 \leq \beta_{i j} \leq 1, \quad(i, j) \in V$ and of $W_{0}$

\section{Proof}

From section 3, eq. (44) and (45) we have

$$
\begin{aligned}
& M=P\left(I-P^{-1}\left(E_{\beta}+G\right)\right)\left(I-P^{-1}\left(F_{\beta}+H\right)\right) \\
& \left.N=\left(E-E_{\beta}\right)+\left(F-F_{\beta}\right)+\left[\operatorname{off}-\operatorname{diag}\left(E_{\beta}+G\right) P^{-1}\left(F_{\beta}+H\right)\right)-(G+H)\right]
\end{aligned}
$$

and, since

$$
(G+H)=\operatorname{rest}_{W}\left(\left(E_{\beta}+G\right) P^{-1}\left(E_{\beta}+H\right)\right)
$$

it is seen from lemma 3 that $N \geq 0$ under the assumptions of the theorem. The remaining part of the proof is similar to that of theorem 3 and will not be reproduced. 


\subsection{The Oliphant-Buleev-Woznicki method}

Lemma 4

Let $A=K-L-U$ where $K \geq 0, L \geq 0$ and $U \geq 0$ are respectively. diagonal, strictly lower and upper triangular matrices and let the diagonal, strictly lower and upper triangular matrices $P, G$ and $H$ respectively be defined by

$$
\begin{aligned}
& \left.P=(I+\alpha) K-\operatorname{diag}\left[(L+G) P^{-1}(U+H) S\right)\right] \\
& G+H=\operatorname{rest}_{W}\left((L+G) P^{-1}(U+H)\right.
\end{aligned}
$$

where $\alpha$ is a diagonal matrix of parameters, $S$ is the matrix whose all entries are equal to 1 and $W$ is a given set of off-diagonal entries (deleting zero entries).

Moreover let $\alpha^{\circ}, \mathrm{P}^{\mathrm{O}}, \mathrm{G}^{\mathrm{O}}$ and $\mathrm{H}^{\mathrm{O}}$ be defined by the same relations together with the additional condition

$$
\alpha^{\mathrm{O}}=\mathrm{K}^{-1}\left[\operatorname{diag}\left(\left(\mathrm{L}+\mathrm{G}^{\mathrm{O}}\right)\left(\mathrm{P}^{\mathrm{O}}\right)^{-1}\left(\mathrm{U}+\mathrm{H}^{\mathrm{O}}\right) \mathrm{S}\right)-\operatorname{diag}\left(\left(\mathrm{L}+\mathrm{G}^{\mathrm{O}}\right)\left(\mathrm{P}^{\mathrm{O}}\right)^{-1}\left(\mathrm{U}+\mathrm{H}^{\mathrm{O}}\right)\right)\right]
$$

and $\alpha_{\mathrm{s}}^{\mathrm{o}}=0$, where $\alpha^{\circ}=\left(\alpha_{\mathrm{i}}^{\circ}\right)$.

If $\mathrm{A}$ is an M-matrix and $\alpha \geq \alpha^{\circ}, \mathrm{P}$ is positive, $\mathrm{G}$ and $\mathrm{H}$ are nonnegative. Moreover $P$ is a non-decreasing function of $A$, an increasing function of $\alpha$ and $a$ non-increasing function of $W ; G$ and $H$ are non-increasing function of $A$ and of $\alpha$ and $G+H$ is an increasing function of $W$.

\section{Proof}

The last parts of the lemma are proven by induction, provided $P>0$ : But for $\alpha=\alpha^{\circ}, \mathrm{P}$ is positive by lemma 3. The proposition follows. Coro11ary

If $A$ is an M-matrix, $0 \leq \beta_{i j} \leq 1$ for $(i, j) \in V$ and $\alpha \geq \alpha^{\circ}$, then the diagonal matrix $P$ of the Oliphant-Buleev-Woznicki method is positive while the off-diagonal matrix $G+H$ is non-negative. Moreover $P$ is a non-increasing function of $\beta_{i j}$ for $0 \leq \beta_{i j} \leq 1,(i, j) \in V$ an increasing function of $\alpha$ and 
a non-increasing function of $W ; G$ and $H$ are non-decreasing functions of $\beta_{i j j}$ for $0 \leq \beta_{i j} \leq 1,(i, j) \in V$, and non-increasing functions of $\alpha$ and $\mathrm{G}+\mathrm{H}$ is an increasing function of $\mathrm{W}$.

\section{Theorem 6}

If $A$ is an M-matrix, $0 \leq \beta_{i j} \leq 1$ for $(i, j) \in V$ and $\alpha \geq \alpha^{\circ}$, then the Oliphant-Buleev-Woznicki method is convergent.

If $T=M^{-1} N$ is the associated iteration matrix, $\rho(T)$ is a nonincreasing function of $\beta_{i j}$ for $0 \leq \beta_{i j} \leq 1$, (i,j) $\in V$, a non-decreasing function of $\alpha$ for $\alpha \geq \alpha^{\circ}$ and a non-increasing function of $W$.

If moreover $A^{-1}>0, \rho(T)$ is a decreasing function of $\beta_{i j}$ for $0 \leq \beta_{i j} \leq 1,(i, j) \in V$, an increasing function of $\alpha$ for $\alpha \geq \alpha^{\circ}$ and a decreasing function of $W$.

Proof

The proof is similar to that of theorem 4 and will not be reproduced. 


\section{Classification of block-factorization iterative methods}

In this section and in the next one, all matrices are assumed to be partitioned into blocks according to one and the same partitioning.

\subsection{Basic methods}

The generalization of the properties of the Buleev method to block factorization processes is not straightforward and will be considered in a companion report [167; we consider here the block 01iphant and Woznicki methods.

\subsection{The block Oliphant method}

Using the notation of section 2, the block 01iphant method is defined by

$$
\begin{aligned}
& E_{1}=E_{\beta} \quad F_{1}=F_{\beta} \\
& P=D-\text { block-diag }\left(E_{\beta} P^{-I_{F}}\right) \\
& E_{\beta}=-\left(B_{i s} \cdot A_{i s}\right) \quad(i, s) \in V_{E} \\
& F_{\beta}=-\left(\beta_{s j} \cdot A_{s j}\right) \quad(s, j) \in V_{F}
\end{aligned}
$$

where $A_{i j}, i, j=1,2 \ldots n$, are the block-entries of $A, V_{E}$ and $V_{F}$ are the sets of couples of indices which correspond to non-zero block-entries of $E$ and $F$ respectively; $\beta_{i j}$ are blocks of arbitrary parameters defined for $(i, j) \in V$ the set of non-zero off-block-diagonal block-entries of $A$ and B.C denotes the element by element product. It is assumed that $\beta_{i j}$ has the same dimensions and the same zero entries as the corresponding block of A.

The corresponding splitting is given by

$$
\begin{aligned}
& \left.M=P-E_{\beta}\right) P^{-1}\left(P-F_{\beta}\right) \\
& N=\left(E-E_{\beta}\right)+\left(F-F_{\beta}\right)+\text { block-offdiag }\left(E_{\beta} P^{-1} F_{\beta}\right)
\end{aligned}
$$

\subsection{The block Woznicki method}

The block Woznicki method is defined by

$$
E_{1}=E+G \quad F_{1}=F+H
$$




$$
\begin{aligned}
& P=D-\operatorname{block-diag}(E+G) P^{-1}(F+H) \\
& G+H=\operatorname{rest}_{W}\left((E+G) P^{-1}(F+H)\right)
\end{aligned}
$$

where $G$ and $H$ are respectively strictly lower and upper block-triangular matrices and $W$ is an arbitrary set of off-diagonal block-entries (deleting zero block-entries).

\subsection{The block Oliphant-Woznicki method}

The block 0liphant-Woxnicki method is define, with obvious notations by

$$
\begin{aligned}
E_{1} & =E_{B}+G \quad F_{1}=F_{\beta}+H \\
P & =D-b l o c k-d i a g\left(\left(E_{\beta}+G\right) P^{-1}\left(F_{\beta}+H\right)\right) \\
G & +H=\operatorname{rest}_{W}\left(\left(E_{\beta}+G\right) P^{-1}\left(F_{\beta}+H\right)\right) \\
E_{\beta} & =-\left(\beta_{i s} \cdot A_{i s}\right),(i, s) \in V_{E} \\
F_{\beta} & =-\left(\beta_{s j} \cdot A_{s j}\right),(s, j) \in V_{F} \\
M & =\left(P-E_{\beta}-G\right) P^{-1}\left(P-E_{\beta}-H\right) \\
N=\left(E-E_{\beta}\right) & +\left(F-F_{\beta}\right)+\left[b l o c k-o f f d i a g\left(\left(E_{\beta}+G\right) P^{-1}\left(F_{\beta}+H\right)\right)-(G+H)\right]
\end{aligned}
$$


6 Eactorizability Conditions and Convergence Analys is of Block-Methods for M-Matrices

6.1 The b lock Oliphant method

Generalizing the analysis given for the point factorization iterative methods, we shall investigate conditions which insure that $P$ is an M-matrix (i.e., positive for the point partitioning) and deduce, as a consequence, convergence rate comparisons.

Lemma 5

Let $\mathrm{A}=\mathrm{K}-\mathrm{L}-\mathrm{U}$ where $\mathrm{K}:, \mathrm{L} \geq 0$ and $\mathrm{U} \geq 0$ are respectively blockdiagonal, strictly lower and upper block-triangular matrices and $\mathrm{K}$ is essentially non-positive. Let the block-diagonal matrix $P$ be defined by

$$
P=k-\text { block-diag }\left(L P^{-1} U\right)
$$

If $A$ is an M-matrix, $P$ is an M-matrix; moreover $P$ is a non-decreasing function of A.

Proof.

Let $P_{i i}, K_{i i}, L_{i s}$ and $U_{s i}$ denote the block-entries of the partitioned matrices $P, K, I$ and $U$ respectively.

Since $A$ is an M-matrix, there exists a vector $X \geq 0$ such that $A X>0$. We prove by induction that the sth "block-component" of the partitioned vector $(\mathrm{P}-\mathrm{U}) \mathrm{X}$ is positive and $\mathrm{P}_{\mathrm{SS}}$ is an M-matrix.

This proposition is evident for $s=1$ since $((P-U) X)_{1}=(A X)_{1}>0$ and $P_{11}=K_{11}$ in an M-matrix (see comments of theorem 1 ).

Assume that $((P-U) X)_{t}>0$ and that $P_{t t}$ is an M-matrix for $t \leq s-1$; then $\mathrm{P}_{t t}^{-1} \geq 0$, thus also $\mathrm{L}_{s t} \mathrm{P}_{t \mathrm{t}}^{-1} \mathrm{U}_{t s} \geq 0$ and therefore the off-diagona1 elements of $P_{s s}$ which is defined by

$$
P_{s s}=K_{s s}-\sum_{t=1}^{s-1} I_{s t} P_{t t}^{-1} U_{t s}
$$


are non-positive. Moreover we have

$$
((P-U) X)_{s}=K_{s s} X_{s}-\sum_{t=1}^{s-1} L_{s t} P_{t t}^{-1} U_{t s} X_{s}-\sum_{j=s+1}^{n} U_{s j} X_{j}
$$

But, from the induction hypothesis,

$$
P_{t t} X_{t}>\sum_{j=t+1}^{n} U_{t j} X_{j} \geq U_{t s} X_{s} \geq 0 \text { and } P_{t t}^{-1} \geq 0
$$

Therefore,

$$
\begin{aligned}
& X_{t} \geq P_{t t}^{-1} U_{t s} X_{s} \\
& L_{s t} X_{t} \geq L_{s t} P_{t t}^{-1} U_{t s} X_{s}
\end{aligned}
$$

and

$$
((P-U) X)_{s} \geq K_{s s} X_{s}-\sum_{t=1}^{s-1} L_{s t} X_{t}-\sum_{j=s+1}^{n} U_{s j} X_{j}=(A X)_{s}>0
$$

This implies that

$$
P_{s s} X_{s}>0
$$

Thus $P_{\text {sS }}$ has non-positive off-diagonal elements and there exists $X_{s} \geq 0$ such that $P_{s s} X_{s}>0$; then, by theorem $1, P_{s s}$ is an M-matrix.

For the second part of the lemma, assume with obvious notations that $A^{\prime}=K^{\prime}-L^{\prime}-U^{\prime}$ with $K^{\prime}$ essentially non-positive, $L^{\prime} \geq 0$, and $U^{*} \geq 0$ is such that $A^{\circ} \geq A$.

Sirice these conditions imply that $A^{\prime}$ is an M-matrix, $P^{\prime}$ is also an M-matrix by applying the first part of the lemma, and so are its block components $\mathrm{P}^{\prime} \mathrm{ss}^{\circ}$

On the other hand we have

$$
\mathrm{P}_{112}^{\prime}=\mathrm{K}_{11}^{\prime} \geq \mathrm{K}_{11}=\mathrm{P}_{11}
$$

and, assuming $P_{t t}^{\prime} \geq P_{t t}$ for $t \leq s-1$, we have since $P_{t t}$ and $P_{t t}^{\prime}$ are M-matrices

$$
P_{t t}^{-1} \geq P_{t t}^{-1}
$$


Therefore

$$
P_{s s}^{\prime}=K_{s s}^{\prime}-\sum_{t=1}^{s-1} L_{s t}^{\prime} P_{t t}^{-1} U_{t s}^{\prime} \geq R_{s s}-\sum_{t=1}^{s-1} L_{s t} P_{t t}^{-1} U_{t s}=P_{s s}
$$

An immediate consequence is:

\section{Corollary}

If $A$ is an M-matrix and the entries of $\beta_{i j}$ for $(i, j) \in V$ belong to $[0,1]$, then the block-diagonal matrix $P$ of the block oliphant method is an M-matrix.

Moreover $P$ is a non-increasing function of $\beta_{i j},(i, j) \in V$ in the range.

Theorem 7

If $A$ is an M-matrix and the entries of $\beta_{i j},(i, j) \in V$ belong to $[0,1]$, then the block Oliphant method is convergent.

If $\mathrm{T}=\mathrm{M}^{-1} \mathrm{~N}$ is the associated iteration matrix, $\rho(\mathrm{T})$ is a non-increasing function of $\beta_{i j},(i, j) \in V$ in this range.

If moreover $A^{-1}>0$ and $D_{S S}^{-1}>0$ for $s=1,2 \ldots n$, where $D$ is the block-diagonal of $A$, then $\rho(T)$ is a decreasing function of the blocks $\beta_{i j},(i, j) \in V$ in this range.

Proof

Similarly as in the proof of theorem 3, but operating with blockmatrices, we have

$$
\begin{aligned}
& M^{-1}=\left(I-P^{-1} F_{\beta}\right)^{-1}\left(I-P^{-1} E_{\beta}\right)^{-1} P^{-1} \\
& N=\left(E-E_{\beta}\right)+\left(F-F_{\beta}\right)+b l o c k-o f f d i a g\left(E_{\beta} P^{-1} F_{\beta}\right) \\
& \left(I-P^{-1} F_{\beta}\right)^{-1}=\sum_{k=0}^{n}\left(P^{-1} F_{\beta}\right)^{k} \\
& \left(I-P^{-1} E_{\beta}\right)^{-1}=\sum_{k=0}^{n}\left(P^{-1} E_{B}\right)^{k}
\end{aligned}
$$

so that $M^{-1} \geq 0$ and $N \geq 0$ from lema 1 when the entries of $\xi_{i j}(i, j) \in V$ 
belong to $[0,1]$.

Thus the splitting $A=M-N$ is regular and, since $A$ is an M-matrix, is convergent.

On the other hand, assuming

$$
\beta_{i j} \leq \beta_{i j}^{\prime} \text { for all }(i, j) \in V \text {, equality excluded, }
$$

we have

$$
\begin{aligned}
& E_{B^{\prime}} \geq E_{B} \\
& F_{B}, \geq F_{B}
\end{aligned}
$$

and equality is excluded for at least one of these inequalities.

From lemma 5 we have also

$$
\mathrm{P}^{-1} \geq \mathrm{P}^{-1} \geq 0
$$

and, using expansion (163) and (164),

$$
\begin{gathered}
M^{-1} \geq M^{-1} \\
\text { If, moreover, } \mathrm{K}_{\mathrm{ss}}^{-1}>0 \text { for } \mathrm{s}=1,2 \ldots \ldots \mathrm{n} \text {, then, from } \\
\mathrm{P}_{\mathrm{SS}} \leqslant \mathrm{K}_{\mathrm{SS}} \text { and } \mathrm{P}_{\mathrm{sS}}^{-1} \geq 0
\end{gathered}
$$

we conclude that

$$
\mathrm{P}_{\mathrm{sS}}^{-1} \geq \mathrm{K}_{\mathrm{sS}}^{-1}
$$

thus

$$
\mathrm{P}_{\mathrm{sS}}^{-1} \geq \mathrm{P}_{\mathrm{sS}}^{-1}>0
$$

Then, it is seen from (166) and (167) that

$$
\begin{aligned}
& \mathrm{P}^{-1} \mathrm{E}_{\beta}, \geq \mathrm{P}^{-1} \mathrm{E}_{\beta} \\
& \mathrm{P}^{-1} \mathrm{~F}_{\beta}, \geq \mathrm{P}^{-1} \mathrm{~F}_{\beta}
\end{aligned}
$$

with equality excluded for at least one of these inequalities. Thus, using expressions (163) and (164)

$$
M^{-1} \geq M^{-1} \text { equality excluded }
$$

The conclusions follow by applying theorem 2 . 


\subsection{The block Oliphant-Woznicki method}

$\underline{\text { Lemma } 6}$

Let $A=K-L-U$ where $K, L \geq 0$ and $U \geq 0$ are respectively blockdiagonal, strictly lower and upper block-triangular matrices and $\mathrm{R}$ is essentially non-positive and let the block-diagonal, strictly lower and upper block-triangular matrices $P, G$ and $H$ respectively be defined by

$$
\begin{aligned}
& P=K-b \text { lock-diag }\left((L+G) P^{-1}(U+H)\right) \\
& G+H=\operatorname{rest}_{W}\left((L+G) P^{-1}(U+H)\right)
\end{aligned}
$$

where $W$ is a given set of off-diagonal block-entries (deleting zero entries).

If $A$ is an M-matrix, $P$ is an M-matrix, $G$ and $H$ are non-negative matrices; moreover $P$ is a non-decreasing function of $A$ and a non-increasing function of $W ; G$ and $H$ are non-increasing functions of $A$ and $G+H$ is an increasing function of $W$.

\section{Proof}

The last parts of the lemma easily follow by induction under the assumption that $P$ is an $M$-matrix (and therefore $G$ and $H$ non-negative). It is thus sufficient to prove that $P$ is an M-matrix when $W$ is maximal or equivalently when $W$ is the complete set of off-blockdiagonal entries.

Letting $\mathrm{P}_{i i}, \mathrm{~K}_{i i}, \mathrm{~L}_{i s}, \mathrm{G}_{i s}, \mathrm{U}_{\mathrm{sj}}$ and $\mathrm{H}_{s j}$ denote the block-entries of the partitioned matrices, $P, K, L, G, U$ and $H$ respectively, the relations defining $P, G$ and $H$ may then be written

$$
\begin{aligned}
& P_{i i}=k_{i i}-\sum_{s=1}^{i-1}\left(I_{i s}+G_{i s}\right) P_{s s}^{-1}\left(U_{s i}+H_{s i}\right) \\
& G_{k i}=\sum_{s=1}^{i-1}\left(I_{k s}+G_{k s}\right) P_{s s}^{-1}\left(U_{s i}+H_{s i}\right) \\
& H_{i k}=\sum_{s=1}^{i-1}\left(I_{i s}+G_{i s}\right) P_{s s}^{-1}\left(U_{s k}+H_{s k}\right) i
\end{aligned}
$$


We prove by induction the following set of relations

$$
\begin{aligned}
& G_{t s} \geq 0 \text { and } H_{s t} \geq 0 \text { for } t>s \\
& ((P-U-H) X)_{S}>0 \text { and } P_{S S} \text { is an M-matrix }
\end{aligned}
$$

where $\mathrm{X}$ is a vector such that $\mathrm{X} \geqslant 0$ and $\mathrm{AX}>0$ and the subscript $\mathrm{s}$ denotes the sth block-component according to the given matrix partitioning.

These relations are evident for $s=1$; assuming that they hold for $s \leq i-1$, we have

$$
G_{k i} \geq 0 \text { and } H_{i k} \geq 0 \text { for } k>i
$$

from the relations $(179),(180)$, since $\mathrm{P}_{\mathrm{ss}}^{-1} \& 0$.

Similarly, from (178) it is seen that the off-diagonal elements of $\mathrm{P}_{i i}$ are non-positive.

As in the proof of lemma 3, we have then

$$
((P-U-H) X)_{i}=K_{i i} X_{i}-\sum_{s=1}^{i-1} \sum_{k=i}^{n}\left(L_{i s}+G_{i s}\right) P_{s s}^{-1}\left(U_{s k}+H_{s k}\right) X_{k}-\sum_{k=i+1}^{n} U_{i k} X_{k}
$$

and, defining the sequence of vectors $\alpha_{s}^{p}$ by the recursive relation

$$
\alpha_{s}^{p}=\sum_{k=s+1}^{i-p} P_{s s}^{-1}\left(U_{s k}+H_{s k}\right) \alpha_{k}^{(p-1)}
$$

for $p>0$ with

$$
\alpha_{s}^{0}=\sum_{k=i}^{n} P_{s s}^{-1}\left(U_{s k}+H_{s k}\right) x_{k}
$$

it is seen that

$$
\sum_{s=1}^{i-1}\left(L_{i s}+G_{i s}\right) \alpha_{s}^{0}=\sum_{s=1}^{i-1} \sum_{p=0}^{i-s-1} L_{i s} \alpha_{s}^{p}
$$

Similarly, it is proven (by induction on q) that

$$
\sum_{p=0}^{q} \alpha_{s}^{p}<x_{s}
$$

and therefore 


$$
\begin{aligned}
((P-U-H) X)_{i} & =k_{i i} x_{i}-\sum_{s=1}^{i-1} L_{i s}\left(\sum_{p=0}^{i-s-1} \alpha_{s}^{p}\right)-\sum_{k=1+1}^{n} U_{i k} x_{k} \\
& \geq k_{i i} x_{i}-\sum_{s=1}^{i-1} L_{i s} x_{s}-\sum_{k=i+1}^{n} U_{i k} x_{k}=(A X)_{i}>0 .
\end{aligned}
$$

Thus also

$$
\mathbf{P}_{\text {ii }} \mathrm{X}_{i}>0
$$

which proves by theorem 1 that $P_{i i}$ is an M-matrix.

\section{Corollary}

If $A$ is an M-matrix and the entries of $B_{1 j},(i, j) \in V$ belong to $[0,1]$, then the block-diagonal matrix. $P$ of the block Oliphant-Woznicki method is an M-matrix while the off block-diagonal matrix $\mathrm{G}+\mathrm{H}$ is non-negative.

Moreover, $P$ is a non-increasing function of $\beta_{i j}$ for $(i, j) \in V$ in this range, and of $W ; G$ and $H$ are non-decreasing functions of $\beta_{i j},(i, j) \in V$ in this range and $G+H$ is an increasing function of $W$.

\section{Theorem 8}

If $A$ is an $M$-matrix and the entries of $\beta_{i j},(1, j) \in V$ belong to $[0,1]$, then the block 0liphant-Woznicki method is convergent.

If $T=M^{-1} N$ is the associated iteration matrix, $\rho(T)$ is a non-increasing function of $\beta_{i j},(i, j) \in V$ in this range as well as a non-increasing $b$ function of $w$.

If moreover $A^{-1}>0$ and $D_{s s}^{-1}>0$ for $s=1,2 \ldots$ where $D$ is the block-diagonal of $A$, then $\rho(T)$ is a decreasing function of $\beta_{i j},(i, j) \in V$, in this range and a decreasing function of $W$.

\section{Proof}

The proof is similar to that of theorem 7 and will not be reproduced. 
7. Concluding Remarks

In this report, we have extended to the class of M-matrices the theory which was developed in [8] for essentially non-positive and either strictly or irreducibly diagonally dominant matrices. Some preliminary results were presented in [11]. Moreover, we have introduced some families of block-factorization iterative methods and extended the theory for M-matrices to these block-methods.

Useful complements to this work would be the numerical experimentation of block factorization iterative methods and the comparison between point and block methods.

For the latter point, some particular cases have been considered in $\lceil 10\rceil$, when the block-factorization method reduces to block Jacobi or block Gauss-Seidel; the general case is however an open question.

For the first point, it may be worthwhile to note that, excepting similar particular cases, it will be necessary to compute the inverse of the block-diagonal matrix P. Therefore, it is felt that for practical applications, the block-size will usually be kept rather small.

\section{Acknowledgement}

We express here our gratitude to Professors E. H. Bareiss and I. K. Abu-Shumays for their numerous and helpful suggestions. 


\section{References}

1. N. I. Buleev, "A numerical method for the solution of two-dimensional and three-dimensional equations of diffusion", Mat. Sb., 51 227-238 (1960) Russian.

2. T. A. Oliphant, "An implicit numerical method for solving twodimensional time-dependent diffusion problems", Quart. Appl. Math., 19, 221-229 (1961).

3. T. A. Oliphant, "An extrapolation process for solving linear systems", Quart. App1. Math, 20, 257-267 (1962).

4. H. L. Stone, "Iterative solution of implicit approximations of multidimensional partial differential equations", SIAM J. Numer. Ana1., 5, 530-558 (1968).

5. T. Dupont, R. P. Kenda11 and H. H. Rachford, "An approximate factorization procedure for solving self-adjoint elliptic difference equations", SIAM J. Numer. Ana1., 5, 559-573 (1968).

6. T. Dupont, "A factorization procedure for the solution of elliptic difference equations", SIAM J. Numer.Anal., $5,753-782$ (1968).

7. Z. Woznicki, "Two-sweep iterative methods for solving large linear systems and their application to the numerical solution of multi-group multi-dimensional neutron diffusion equation", Ph.D. Thesis, Institute of Nuclear Research, Swierk (1973).

8. R. Beauwens, "On the analysis of factorization procedures", Internal Report, Universite Libre de Bruxelles (1973)。

9. R. Beauwens and M. Borysiewicz, "On the convergence of block-iterative and factorization iterative methods for symmetric or unsymmetric complex matrices", Internal Report, Universite Libre de Bruxelles (1973).

10. R. Beauwens, "An order relation between factorization iterative methods", Internal Report, Universite Libre de Bruxelles (1973).

11. Ro Beauwens, "Factorization iterative methods for M-matrices", Unpublished report. (1973).

12. R. S. Varga, "Matrix iterative analysis", Prentice Hal1 (1962).

13. K. Fan, "Topological proofs for certain theorems on matrices with non-negative elements", Monatsh - Math, 62, 219-237 (1958).

14. M. Fiedler and V. Ptak, "Some generalizations of positive definiteness and monotonicity", Numer. Math., 2, 163-172 (1966).

15. Lo Collatz, "Aufgaben monotoner Art", Arch. Math.s 3, 366-376 (1952).

16. R. Beauwens, "On the point and block factorization iterative methods for arbitrary matrices and the characterization of M-matrices", submitted for pubifcation in the Series in App1. Math., Northwestern University, Evanston, IL (1973). 